\title{
The Diagnosis of Some Lung Diseases in a Prolog Expert System
}

\author{
Jimmy Singla \\ Asst. Prof. \\ North West Institute of Engg. \& \\ Tech. Dhudike \\ Punjab Technical University \\ Jalandhar
}

\begin{abstract}
This medical expert system is used to diagnose the main lung diseases among the patients. The diagnosis is made taking into account the symptoms that can be seen or felt. This medical expert system helps the doctor or expert in making the appropriate diagnosis of the patient. The lung diseases have many common symptoms and some of them are very much alike. This creates many difficulties for the lungs doctor to reach at a right decision or diagnosis. This expert system can remove these difficulties and it is having knowledge of thirtytwo lung diseases. This expert system is implemented in SWIProlog.
\end{abstract}

\section{General Terms}

Computer Science, Artificial Intelligence, Expert System

\section{Keywords}

Diagnosis, Symptoms, Knowledge Base, Facts

\section{INTRODUCTION}

Some people call Computer Science as Computing Science. It is abbreviated as CS. As its name suggests, it is practical approach to computation. A computer scientist is the one who specializes in the theory of computation and the design of computational system. Computer science has large number of theoretical and practical disciplines. Examples of these disciplines are computational complexity theory, computer graphics, computer programming etc. The main areas of computer science are divided in to two categories. These two categories are Theoretical Computer Science and Applied Computer Science. Theoretical Computer Science includes theory of computation (TOC), information and coding theory, algorithms and data structures (Algo. \& DS), programming language (PL) theory. Applied Computer Science includes artificial intelligence (AI), computer architecture (CA) and engineering, computer graphics (CG) and visualization, computer security and cryptography, computer networks $(\mathrm{CN})$, distributed systems (DS), database and information retrieval, software engineering (SE).

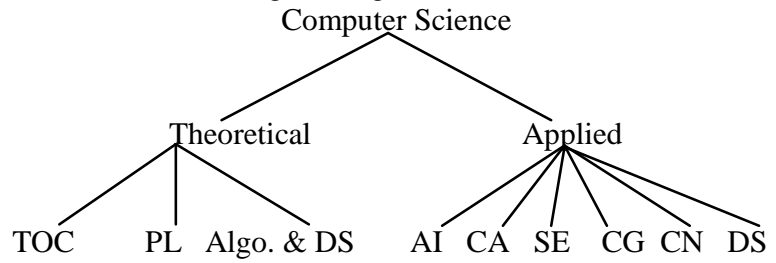

Fig.1: Areas of Computer Science

\subsection{Artificial Intelligence}

Artificial intelligence is a branch of computer science that deals with intelligent machines and software. It mainly concerns with deep study and development phases of intelligent machines. Some authors also define Artificial Intelligence as "The study and design of intelligent agents, where intelligent agent is a system that perceives its environment and takes actions that maximize its chances of success". Artificial intelligence systems are used in economics, medicines and military. These systems also have many software applications like video games, computer chess etc. The goals of artificial intelligence research are reasoning, knowledge, planning, learning, communication, perception and ability to manipulate objects. Artificial intelligence is divided into two categories. These two categories are conventional artificial intelligence and computational intelligence. Conventional artificial intelligence includes machine learning and stastical analysis. Computational intelligence includes neural networks and fuzzy systems

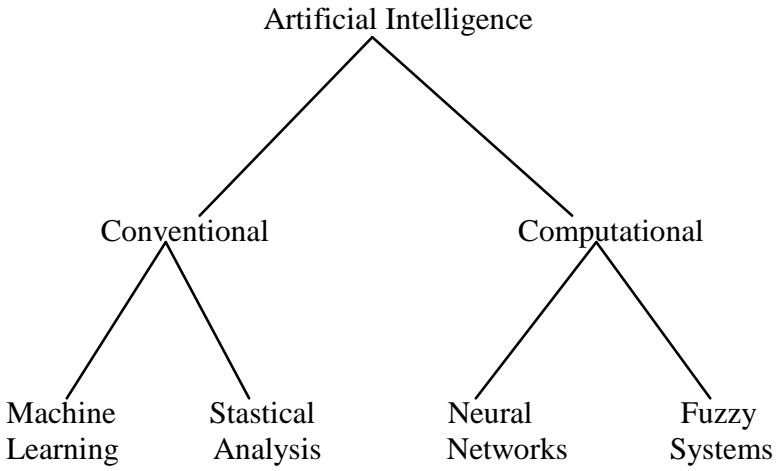

Fig. 2: Areas of Artificial Intelligence

The other applications of artificial intelligence are Automation, Computer Vision, Artificial Creativity, Expert System and Knowledge Management.

\subsection{Expert System}

An expert system is a set of programs that manipulate knowledge to solve problems in a specialized domain that requires human expertise. An expert system is also called knowledge based system. It is a computer program that contains some of the subject specific knowledge of one or more human experts. The main components of expert system are knowledge base and inference engine. Knowledge base contains the domain knowledge needed to solve the problems in the form of rules. The rules are a popular paradigm for representing knowledge. Inference engine is the code at the core of the system which derives conclusions from knowledge 
base through inference or reasoning. The major features of expert system are user interface, data representation, inference, explanations, coping with uncertainty and advantages of expert system are fast response, increased reliability, reduced cost, reducing errors, multiple expertise, intelligent database, reduced danger. There are also some disadvantages of expert system. Disadvantages are absence of common sense, no response in exceptional cases, and no change with changing environment.

\subsection{Medical Expert System}

A large number of expert systems are medical. The main aim of any medical expert system is diagnosis and treatment of diseases. A medical expert system is built up of programs and medical knowledge base. The information obtained from medical expert system is similar to the information given by doctor or expert in that particular area. Our medical expert system has thirty-two lung diseases in its knowledge base. The user or patient is asked to answer with YES or NO, If a particular symptom appears or not. In the end, based on user's or patient's answers, the name of the disease is displayed on the screen. A limitation of this medical expert system is that only symptoms entered by the programmer in the knowledge base are available. It does not think and learn by itself. Therefore the knowledge base can be updated any time with new symptoms and new diseases.

\section{PROPOSED STRUCTURE}

A Rule based expert system has the following components-

1) The Knowledge Base contains information about thirty-two lung diseases which are represented as a set of if-then production rules. The knowledge base is analogue to the long term human memory. The total ordering of production rules is done in the knowledge base.

You may understand it with the help of examples. Tuberculosis is a lung disease whose symptoms are persistant cough, constant fatigue, weight loss, loss of appetite, fever, coughing up blood, night sweats. So it will be stored in knowledge base in the form of a rule which is as follow:-

Disease (Patient, tuberculosis):-

Symptom (Patient, persistant_cough),

Symptom (Patient, constant_fatigue),

Symptom (Patient, weight_loss),

Symptom (Patient, loss_of_appetite),

Symptom (Patient, fever),

Symptom (Patient, coughing_up_blood),

Symptom (Patient, night_sweats).

You can also take some other examples, which are given below:-

Pneumonia is a disease whose symptoms are cough, fever, shaking chills, shortness of breath. So it will be stored in knowledge base as follow:-

Disease (Patient, pneumonia):-

Symptom (Patient, cough),

Symptom (Patient, fever),

Symptom (Patient, shaking_chills),

Symptom (Patient, shortness_of_breath).

Byssinosis is a disease whose symptoms are chest tightness, cough, wheezing. So it will be stored in knowledge base as follow:-
Disease (Patient, byssinosis):-

Symptom (Patient, chest_tightness),

Symptom (Patient, cough),

Symptom (Patient, wheezing).

Pertusis is a disease whose symptoms are runny nose and mild fever. So it will be stored in the knowledge base as follow:-

Disease(Patient, pertusis):-

Symptom(Patient, runny_nose),

Symptom(Patient, mild_fever).

Pneumoconiosis is a disease whose symptoms are chronic cough and shortness of breath. So it will be stored in the knowledge base as follow:-

Disease (Patient, pneumoconiosis):-

Symptom (Patient, chronic_cough),

Symptom (Patient,shortness_of_breath).

Sarcoidosis is a disease whose symptoms are dry cough, shortness of breath, mild chest pain, scaly rash, fever, red bumps on legs, sore eyes and swollen ankles. So it will be stored in knowledge base as follow:-

Disease (Patient, sarcoidosis):-

Symptom (Patient, dry_cough),

Symptom (Patient, shortness_of_breath),

Symptom (Patient, mild_chest_pain),

Symptom (Patient, scaly_rash),

Symptom (Patient, fever),

Symptom (Patient, red_bumps_on_legs),

Symptom (Patient, sore_eyes),

Symptom (Patient, swollen_ankles).

Similarly in this way we have stored total thirty-two different lung diseases in the knowledge base.

2) The Fact Base contains facts which are used to match against the antecedent part of rules stored in the knowledge base. The fact base is analogue to the short term human memory.

3) The main task of Inference Engine is to carry out the reasoning by linking the rules with facts and deducing new facts.

4) The User Interface is used to communicate between user and expert system.

5) The Explanation Module enables the user to ask the expert system how a particular conclusion is reached and why a specific fact is needed.

6) The Developer Interface is used to modify the knowledge

\section{WORKING OF THE PROGRAM}

In the following figure we present the simulation of the program. 


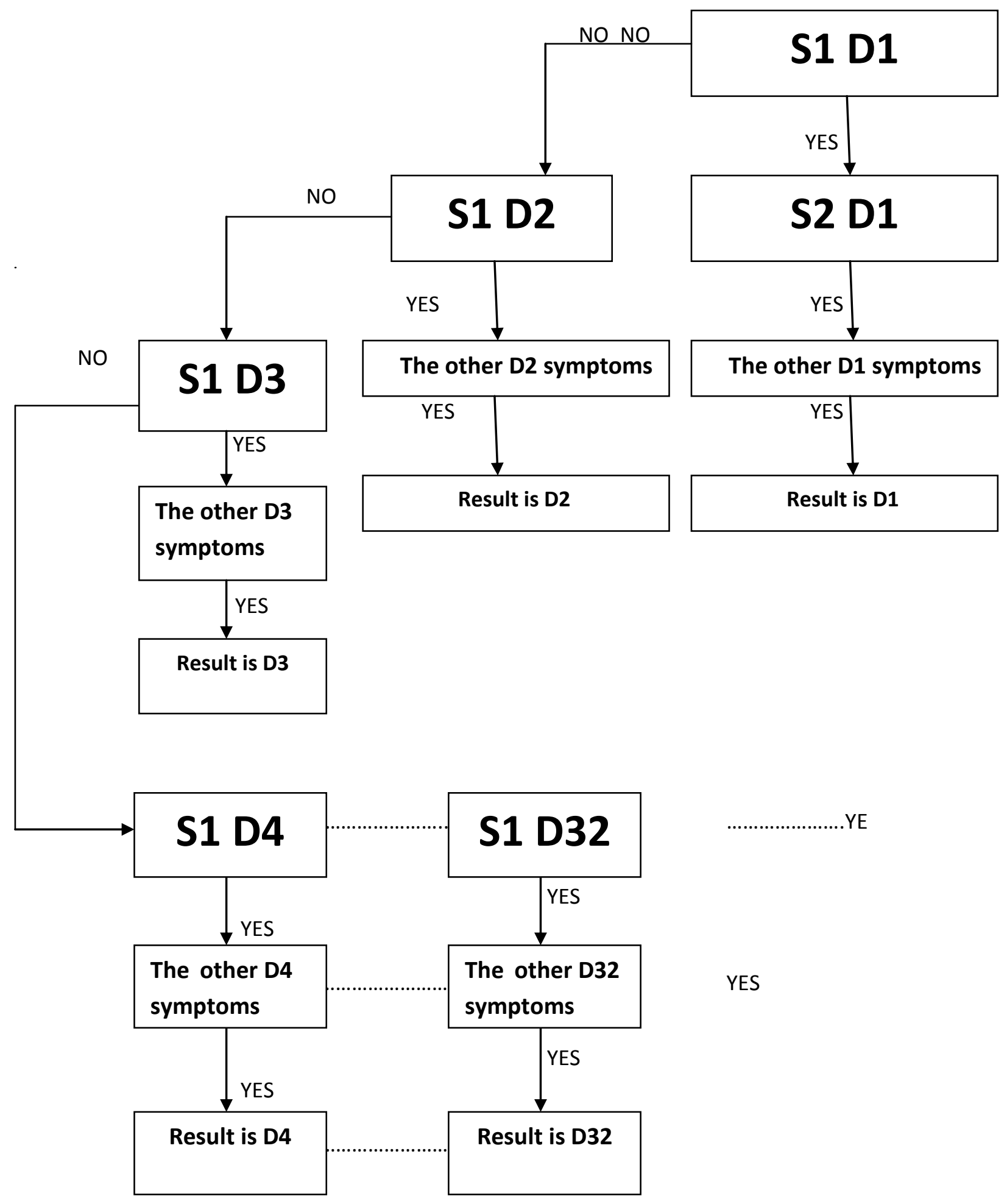

Fig. 3 Working of a Program 
In the above figure, S1 D1 denotes the first symptom of first disease. In general Si Dj denotes the "i" symptom of " $j$ " disease. If the program has a positive answer to the symptom, it goes on with the symptoms from that disease. If only one symptom from that disease is negative, it jumps to the first symptom from the next disease.

\section{RESULTS}

This Prolog expert system is successfully implemented and results are taken. It is applied on many patients and its results are $70 \%$ correct. For example Asthma is a disease whose symptoms are wheezing, cough, chest tightness, shortness of breath. In this Prolog expert system, First the user or patient enters his or her name (suppose name is Jimmy) then the user or patient is asked to answer with YES (y) or NO (n), If a particular symptom appears or not. (Here user or patient will reply YES (y) to the symptoms wheezing, cough, chest tightness, shortness of breath.) In the end, based on user's or patient's answers, the name of the disease is displayed on the screen (Asthma).

\section{CONCLUSIONS}

This medical expert system is dealing with person's health and an approximate diagnosis of a certain disease is established, this system has a great risk. In reality, there may be more lung diseases which are not considered in the system's knowledge base. So, this knowledge base is incomplete but it can be updated any time with new symptoms and diseases. Symptoms already available in knowledge base are not $100 \%$ correct because different doctors have different opinions and there are anomalies in medicines.

\section{ACKNOWLEDGEMENT}

The author wishes to express his sincere gratitude and indebtedness to his parents Er. R. K Singla (SDO) and Mrs. Bindu Singla for their moral and financial support and encouragement without which the author would not have been able to bring out this research work.

\section{REFERENCES}

[1] Weilin, Jian-Xong Tang, 1991. DiagFH: An expert system for diagnosis of fulminant hepatitis. Computer based medical systems: Fourth annual IEEE Systems.

[2] Reghis, M., Roventa, E., 1998. Classical and Fuzzy Concepts in Mathematical Logic and Applications. CRC Press New York.

[3] Russell, S., Norvig, P., 1995. Artificial Intelligence A Modern Approach. Prentice hall.

[4] Ali, S., Chia. P., 1999 Graphical knowledge-based protocols for chest pain management. IEEE computers in cardiology.

[5] Nilsson, U., Maluszynski, J., 2000 Logic Programming and Prolog (2ed.). John Wiley \& Sons Ltd..

[6] Callear, D., 2001 Prolog Programming for students. Thomson Learning.

[7] Bratko, I., 2000 Prolog Programming for Artificial Intelligence. Addison- Wesley.

[8] Roventa, E., Spircu, T., 2009. Management of Knowledge Imperfection in Developing Intelligent Systems. Springer-Verlag.

[9] Luger, G.L., 2002. Artificial Intelligence Structures and Strategies for Complex Problem Solving. 4ed. AddisonWesley.

[10] Negnivitski , M., 2002 . Artificial Intelligence Guide to Intelligent Systems. Addison-Wesley.

[11] Shu-hsien liao., 2005. Expert System methodologies and applications-a decade review from 1995 to 2004 Elsevier ltd.

[12] Roventa, E., 2009. The diagnosis of some kidney diseases in small prolog expert system. Arad.

Jimmy Singla is an Asst. Prof. with the department of Computer Science \& Engg., North West Institute of Engg. \& Tech. Dhudike, Moga, Punjab. He received his B.Tech and M.Tech in Computer Science \& Engg. from Punjab Technical University Jalandhar, Punjab. He is pursuing Ph.D in Computer Science and Engg. from Punjab Technical University Jalandhar, Punjab. His areas of interest are Artificial intelligence, Digital image processing. He works on diagnosis of various medical diseases in Prolog expert system. 